\title{
ISOMORPHIC SUBGRAPHS HAVING MINIMAL INTERSECTIONS
}

\author{
R. C. MULLIN, B. K. ROY and P. J. SCHELLENBERG
}

(Received 22 October 1981)

Communicated by W. D. Wallis

\begin{abstract}
Given a finite graph $H$ and $G$, a subgraph of it, we define $\sigma(G, H)$ to be the largest integer such that every pair of subgraphs of $H$, both isomorphic to $G$, has at least $\sigma(G, H)$ edges in common; furthermore, $R(G, H)$ is defined to be the maximum number of subgraphs of $H$, all isomorphic to $G$, such that any two of them have $\sigma(G, H)$ edges common between them. We are interested in the values of $\sigma(G, H)$ and $R(G, H)$ for general $H$ and $G$. A number of combinatorial problems can be considered as special cases of this question; for example, the classical set-packing problem is equivalent to evaluating $R(G, H)$ where $G$ is a complete subgraph of the complete graph $H$ and $\sigma(G, H)=0$, and the decomposition of $H$ into subgraphs isomorphic to $G$ is equivalent to showing that $\sigma(G, H)=0$ and $R(G, H)=\varepsilon(H) / \varepsilon(G)$ where $\varepsilon(H), \varepsilon(G)$ are the number of edges in $H, G$ respectively.

A result of S. M. Johnson (1962) gives an upper bound for $R(G, H)$ in terms of $\sigma(G, H)$. As a corollary of Johnson's result, we obtain the upper bound of McCarthy and van Rees (1977) for the Cordes problem. The remainder of the paper is a study of $\sigma(G, H)$ and $R(G, H)$ for special classes of graphs; in particular, $H$ is a complete graph and $G$ is, in most instances, a union of disjoint complete subgraphs.
\end{abstract}

\section{Introduction}

Let $H$ be any finite graph and let $G$ be any subgraph of $H$. We are interested in two integer-valued functions of $G$ and $H, \sigma(G, H)$ and $R(G, H)$. The function $\sigma(G, H)$ is defined to be the largest integer such that every pair of subgraphs of $H$, say $G_{1}$ and $G_{2}$, both isomorphic to $G$, has at least $\sigma(G, H)$ edges in common. This means that each pair of such subgraphs has at least $\sigma(G, H)$ common edges and some pair of them has precisely $\sigma(G, H)$ common edges. If the graph $H$

C 1983 Australian Mathematical Society $0263-6115 / 83 \$ A 2.00+0.00$ 
under consideration is clear from the discussion, we will refer to $\sigma(G, H)$ simply as $\sigma(G)$ or, still more simply, as $\sigma$, in the event that there is no ambiguity about the subgraph $G$ either. The function $R(G, H)$ is defined to be the largest integer $r$ such that there exist $r$ subgraphs of $H$, say $G_{1}, G_{2}, \ldots, G_{r}$, each isomorphic to $G$, with the property that each pair of them has $\sigma$ common edges. When appropriate, we will refer to $R(G, H)$ as $R(G)$ or $R$.

It is interesting to note that the classical set-packing problem is a special case of this problem. The object in the packing problem is to determine the maximum number of $k$-subsets of an $n$-set such that any pair of elements from the $n$-set is in at most one of the $k$-subsets. This is equivalent to the determination of $R(G, H)$ where $H$ is the complete graph $K_{n}, G$ is $K_{k}$ and $\sigma=0$.

Cordes $(1978)^{*}$ considered the problem of determining $R(G, H)$ and $\sigma(G, H)$ in the special case where $H$ is the graph $K_{m n}$ (the complete graph on $m n$ vertices) and $G$ is the union of $n$ disjoint complete graphs, each on $m$ vertices. Since then, others have considered this problem, including Mullin and Stanton (1978), McCarthy and van Rees (1977), and Nemeth (1976). Nemeth was the first to consider the more general problem described above.

For any graph $H$, let $\varepsilon(H)$ denote the number of edges in $H$. In Section 2, we observe that a result of Johnson (1962) gives

$$
R(G, H) \leqslant \frac{E(e-\sigma(G, H))}{e^{2}-E \cdot \sigma(G, H)}
$$

where $E=\varepsilon(H)$ and $e=\varepsilon(G)$. This bound gives the upper bound for $R(G, H)$ obtained by McCarthy and van Rees (1977) in the special case where $H=K_{m n}$ and $G$ is the disjoint union of $n K_{m}$ 's.

In Section 3, we consider lower bounds for $R\left(G, K_{2 n}\right)$ where $G$ is the disjoint union of $2 K_{n}$ 's; in Section 4, we study $R\left(G, K_{n}\right)$ where $G$ is the disjoint union of two complete graphs $K_{m}$ and $K_{n-m}$; and in Section 5, we consider $R\left(G, K_{n}\right)$ where $G$ is a double-cone (see Section 5) on $n$ vertices. For $G$ a double-cone on $n$ vertices, where $n \geqslant 8$, it is shown that $\sigma\left(G, K_{n}\right)=8$ and $R\left(G, K_{n}\right)=2$.

We conclude the introduction by describing some notation to be used in later sections. For any graph $G, V(G)$ is the set of vertices of $G$ and $E(G)$ is the set of edges; furthermore, $\nu(G)=|V(G)|$ and $\varepsilon(G)=|E(G)|$.

\section{The Cordes problem}

Though Cordes (1978) originally phrases this problem in the language of combinatorial designs, we will use the graph theoretical language employed in

\footnotetext{
* Though the paper by Cordes was published in 1978, he introduced the problem in 1976.
} 
Section 1. As mentioned in the introduction, Cordes investigated $\sigma(G, H)$ and $R(G, H)$ in the special case where $H$ is a complete graph on $m n$ vertices and $G$ is the union of $n$ disjoint complete subgraphs, each on $m$ vertices. In this special case, we denote $\sigma(G, H)$ by $\sigma\left(m^{n}\right)$ and $R(G, H)$ by $R\left(m^{n}\right)$.

Let us consider the more general situation where $G$ is the union of disjoint complete subgraphs of $H$, but the complete subgraphs need not all have the same cardinality. We begin by looking at $\sigma(G, H)$. The following result (see Hartman, Mullin and Stinson (1980)) plays a significant role in several of the proofs given below.

LEMMA 2.1. For any $b$ non-negative integers $k_{1}, k_{2}, \ldots, k_{b}$, let

$$
\sum_{i=1}^{b} k_{i}=q b+r, \quad \text { where } 0 \leqslant r<b .
$$

If $q+1 \geqslant u$, then

$$
\sum_{i=1}^{b}\left(\begin{array}{c}
k_{i} \\
u
\end{array}\right) \geqslant r\left(\begin{array}{c}
q+1 \\
u
\end{array}\right)+(b-r)\left(\begin{array}{c}
q \\
u
\end{array}\right)
$$

with equality if and only if $r$ of the $k_{i}$ 's are equal to $q+1$ and the remaining $b-r$ are equal to $q$. If $q+1<u$, then

$$
\sum_{i=1}^{b}\left(\begin{array}{c}
k_{i} \\
u
\end{array}\right) \geqslant 0
$$

with equality if and only if each $k_{i}<u$.

We are now in a position to prove

THEOREM 2.2. For $m, n$ and $s$ positive integers such that $m, n \geqslant 1,0 \leqslant s<n$, let $H=K_{t}$ where $t=m n+s$; that is, let $H$ be a complete graph on $t$ vertices. Let $G$ be the union of $n$ disjoint complete subgraphs of $H, s$ of which have $m+1$ vertices and $n-s$ of which have $m$ vertices. Let $m=k n+l$, where $0 \leqslant l<n$. Then

$$
\sigma(G, H)=(\ln +s)\left(\begin{array}{c}
k+1 \\
2
\end{array}\right)+\left(n^{2}-\ln -s\right)\left(\begin{array}{l}
k \\
2
\end{array}\right)
$$

furthermore, whenever two subgraphs isomorphic to $G$, say $G_{1}$ and $G_{2}$, intersect in $\sigma$ edges, then each connected component of $G_{1}$ intersects each connected component of $G_{2}$ in $k$ or $k+1$ vertices.

Proof. Label the connected components of $G_{i}, i \in\{1,2\}$, by $C_{i}^{1}, C_{i}^{2}, \ldots, C_{i}^{n}$. Let $x_{i j}=\nu\left(C_{1}^{i} \cap C_{2}^{j}\right)$. Clearly

$$
\sum_{i, j} x_{i j}=n m+s=k n^{2}+\ln +s .
$$


The number of edges common to both $G_{1}$ and $G_{2}$ is given by

$$
\sum_{i, j}\left(\begin{array}{c}
x_{i j} \\
2
\end{array}\right) \text {. }
$$

We can apply Lemma 2.1 to the $x_{i j}$ 's with $b=n^{2}, q=k, r=\ln +s$ and $u=2$. Thus

$$
\sum_{i, j}\left(\begin{array}{c}
x_{i j} \\
2
\end{array}\right) \geqslant(\ln +s)\left(\begin{array}{c}
k+1 \\
2
\end{array}\right)+\left(n^{2}-\ln -s\right)\left(\begin{array}{c}
k \\
2
\end{array}\right)
$$

with equality if and only if each $x_{i j}$ is either $k+1$ or $k$. We conclude that

$$
\sigma(G, H)=(\ln +s)\left(\begin{array}{c}
k+1 \\
2
\end{array}\right)+\left(n^{2}-\ln -s\right)\left(\begin{array}{l}
k \\
2
\end{array}\right)
$$

and $G_{1}, G_{2}$ have $\sigma$ edges common if and only if each connected component of $G_{1}$ intersects each connected component of $G_{2}$ in $k$ or $k+1$ vertices.

If $s=0$, we have

$$
\sigma(G, H)=n\left[l\left(\begin{array}{c}
k+1 \\
2
\end{array}\right)+(n-l)\left(\begin{array}{l}
k \\
2
\end{array}\right)\right]
$$

which was first established by Cordes (1978).

We now turn our attention to the function $R(G, H)$. The more general graph-theoretic formulation of Cordes' problem permits us to employ an elegant result of S. M. Johnson (1962) to obtain an upper bound for $R(G, H)$. For the sake of completeness, we include the proof of Johnson's Theorem.

As usual, we let $|X|$ denote the cardinality of the set $X$.

THEOREM 2.3 (1962). Let $H$ be a finite set of cardinality $E$ and let $G_{1}, G_{2}, \ldots, G_{\delta}$ be subsets of $H$ such that

(i) $\left|G_{i}\right|=e$ for $1 \leqslant i \leqslant \delta$, and

(ii) $\left|G_{i} \cap G_{j}\right| \leqslant \theta$ for $1 \leqslant i<j \leqslant \delta$.

Then, for $e^{2}>E \theta$,

$$
\delta \leqslant \frac{E(e-\theta)}{e^{2}-E \theta} .
$$

Proof. Let $A$ be the multi-set of set intersections

$$
\left(G_{i} \cap G_{j} \mid 1 \leqslant i<j \leqslant \delta\right) .
$$

Define a bipartite graph, with bipartition $(A, H)$, as follows: a vertex $h \in H$ is joined to a vertex $G_{i} \cap G_{j} \in A$ if and only if the element $h \in H$ is in the intersection $G_{i} \cap G_{j}$. In order to count the edges of this graph, for each $h \in H$, 
we define $k_{h}$ to be the number of $G_{i}$ 's which contain the element $h$. Clearly, the number of edges incident with the vertex set $H$ is

$$
\sum_{h \in H}\left(\begin{array}{c}
k_{h} \\
2
\end{array}\right)
$$

The number of edges incident with the vertex set $A$ is at most $\left(\begin{array}{l}\delta \\ 2\end{array}\right) \theta$ since $\left|G_{i} \cap G_{j}\right| \leqslant \theta$. Hence

$$
\sum_{h \in H}\left(\begin{array}{c}
k_{h} \\
2
\end{array}\right) \leqslant\left(\begin{array}{l}
\delta \\
2
\end{array}\right) \theta
$$

It is immediate from the definition of the $k_{h}$ 's that

$$
\sum_{h \in H} k_{h}=\delta e .
$$

Combining (I) and (II), we have

$$
\sum_{h \in H} k_{h}^{2} \leqslant \delta(\delta-1) \theta+\delta e .
$$

By the Cauchy-Schwarz inequality,

$$
\left(\sum a_{i} b_{i}\right)^{2} \leqslant\left(\sum a_{i}^{2}\right)\left(\sum b_{i}^{2}\right)
$$

applied with $a_{i}=k_{h}$ and $b_{i}=1$, we obtain

$$
\delta^{2} e^{2} \leqslant(\delta(\delta-1) \theta+\delta e) E,
$$

which simplifies to give

$$
\delta \leqslant \frac{E(e-\theta)}{e^{2}-E \theta}
$$

under the hypothesis that $e^{2}>E \theta$.

Johnson also observed that given any upper bound for $\delta$, one can readily obtain a second inequality which must be satisfied. Let $q=[\delta e / E]$ and let $r=\delta e-q E$. Applying Lemma 2.1 with $q=q, r=r, b=E$ and $u=2$, we obtain that

$$
\sum_{h \in H}\left(\begin{array}{c}
k_{h} \\
2
\end{array}\right) \geqslant r\left(\begin{array}{c}
q+1 \\
2
\end{array}\right)+(E-r)\left(\begin{array}{l}
q \\
2
\end{array}\right) .
$$

Combining this with inequality (I) of the above theorem, we obtain

$$
\left(\begin{array}{l}
\delta \\
2
\end{array}\right) \theta \geqslant r q+E\left(\begin{array}{l}
q \\
2
\end{array}\right) \text {. }
$$


Thus, we have established

LEMMA 2.4. Under the hypothesis of Theorem 2.3,

$$
\left(\begin{array}{l}
\delta \\
2
\end{array}\right) \theta \geqslant r q+E\left(\begin{array}{l}
q \\
2
\end{array}\right)
$$

where $q=[\delta e / E]$ and $r=\delta e-q E$.

This result will occasionally permit us to obtain an improved value for $\delta$; for example, if we have an upper bound for $\delta$ and if the inequality of Lemma 2.4 is not satisfied, then $\delta-1$ is a better upper bound.

In the generalized graph-theoretical formulation of the Cordes problem, the graphs $G$ and $H$ are completely determined by their edge-sets $E(G)$ and $E(H)$, respectively. Thus, if we apply Johnson's Theorem to these two edge-sets, we immediately obtain

COROLlary 2.5. Let $H$ be a graph with $E$ edges and let $G$ be a subgraph with $e$ edges. Then for $\sigma=\sigma(G, H)$,

$$
R(G, H) \leqslant \frac{E(e-\sigma)}{e^{2}-E \sigma} .
$$

Combining Theorem 2.2 in the special case when $s=0$ and Corollary 2.5, we obtain the following result of McCarthy and van Rees.

LeMma 2.6. For the classical Cordes problem,

$$
R\left(m^{n}\right) \leqslant \frac{(m n-1)[m(m-1)-k(m+l-n)]}{m(m-1)^{2}-k(m n-1)(m+l-n)}
$$

where $m=k n+l$ and $0 \leqslant l<n$.

Proof. This follows immediately from Corollary 2.5 when we set

$$
E=\left(\begin{array}{c}
n m \\
2
\end{array}\right), \quad e=n\left(\begin{array}{c}
m \\
2
\end{array}\right)
$$

and from Theorem 2.2, with $s=0$,

$$
\sigma\left(m^{n}\right)=n\left[l\left(\begin{array}{c}
k+1 \\
2
\end{array}\right)+(n-l)\left(\begin{array}{l}
k \\
2
\end{array}\right)\right] .
$$

One can also find an upper bound for $R(G, H)$, with $G$ and $H$ as defined in Theorem 2.2, since

$$
E=\left(\begin{array}{c}
n^{2} k+n l+s \\
2
\end{array}\right), \quad e=s\left(\begin{array}{c}
n k+l+1 \\
2
\end{array}\right)+(n-s)\left(\begin{array}{c}
n k+l \\
2
\end{array}\right),
$$

and $\sigma(G, H)$ is as given in Theorem 2.2. 
We now use Lemma 2.4 to establish the following improved upper bound of McCarthy and van Rees (1977).

TheOREM 2.7. $R\left((2 m+1)^{2}\right) \leqslant 4 m+4$, provided $m \geqslant 2$.

Proof. From Lemma 2.6, we get $R\left((2 m+1)^{2}\right) \leqslant 4 m+5$. Now let $\delta=4 m+$ 5. For this case,

$$
E=\left(\begin{array}{c}
4 m+2 \\
2
\end{array}\right)=8 m^{2}+6 m+1, \quad e=2 \cdot\left(\begin{array}{c}
2 m+1 \\
2
\end{array}\right)=4 m^{2}+2 m,
$$

and

$$
\theta=2 \cdot\left[\left(\begin{array}{c}
m \\
2
\end{array}\right)+\left(\begin{array}{c}
m+1 \\
2
\end{array}\right)\right]=2 m^{2} .
$$

Then $q=2 m+1, r=8 m^{2}+2 m-1$, and hence,

$$
\begin{aligned}
r q+E \cdot\left(\begin{array}{l}
q \\
2
\end{array}\right) & =16 m^{4}+36 m^{3}+20 m^{2}+m-1, \\
\left(\begin{array}{l}
\delta \\
2
\end{array}\right) \theta & =16 m^{4}+36 m^{3}+20 m^{2} .
\end{aligned}
$$

Hence $\delta \leqslant 4 m+4$.

When $H=K_{4 m}$ and $G$ is the union of two disjoint $K_{2 m}$ 's, Cordes (1978) established

THEOREM 2.8. $R\left((2 m)^{2}\right) \leqslant 4 m-1$ with equality if and only if there exists $a$ Hadamard matrix of order $4 m$.

\section{Some lower bounds for $R\left((2 m+1)^{2}\right)$}

McCarthy and van Rees (1977) establish a number of results on $R\left((2 m+1)^{2}\right)$.

LEMMA 3.1. $R\left((2 m+1)^{2}\right) \geqslant R\left((2 m)^{2}\right)$.

LEMMA 3.2. $R\left((4 n+1)^{2}\right) \geqslant 2 \min \left[R\left((2 n)^{2}\right), R\left((2 n+1)^{2}\right)\right]+1$.

LEMMA 3.3. $R\left((4 n-1)^{2}\right) \geqslant 2 \min \left[R\left((2 n-1)^{2}\right), R\left((2 n)^{2}\right)\right]+1$.

LEMMA 3.4. $2^{n+1}-1 \leqslant R\left(\left(2^{n}-1\right)^{2}\right) \leqslant 2^{n+1}$ for $n \geqslant 3$.

LEMMA 3.5. If $p^{\alpha}=4 n+1$, where $p$ is a prime, then $4 n+1 \leqslant R\left((2 n+1)^{2}\right) \leqslant$ $4 n+4$. 
McCarthy and van Rees also demonstrate that $R\left(5^{2}\right)=12$ and determine upper and lower bounds for $R\left((2 m+1)^{2}\right)$ for $2 m+1 \in\{7,9,11,13\}$. Cordes (1978) shows that $R\left(3^{2}\right)=10$.

In this section, we show that $R\left((4 m+3)^{2}\right) \geqslant 8 m+7$ where $4 m+3$ is a prime or a power of a prime. Combining this result with Lemma 3.3, we show that $8 m+7 \leqslant R\left((4 m+3)^{2}\right) \leqslant 8 m+8$ for many non-prime powers $4 m+3$.

We proceed, now, using the language of combinatorial designs rather than graphs. Let $V$ be a set of cardinality $m n$. An $\left(m^{n}\right)$-round, $R$, is a partition of $V$ into $n$ sets, called blocks, each of cardinality $m$. The pairs of an $\left(m^{n}\right)$-round $R$ are precisely those pairs $\{a, b\} \subseteq V, a \neq b$, such that, for some block $B \in \Re$, $\{a, b\} \subseteq B$. Then $\sigma\left(m^{n}\right)$ is the largest integer such that any two $\left(m^{n}\right)$-rounds of $B$ have at least $\sigma\left(m^{n}\right)$ common pairs. Also, $R\left(m^{n}\right)$ is the largest integer $r$ such that there exist $r\left(m^{n}\right)$-rounds of $V$, say $\Re_{1}, \Re_{2}, \ldots, \Re_{r}$, with the property that each pair of these rounds have $\sigma\left(\mathrm{m}^{n}\right)$ common pairs. Throughout the remainder of this section, we investigate $R\left((2 m+1)^{2}\right)$.

We now consider the problem of constructing two $\left((2 m+1)^{2}\right)$-rounds having $\sigma\left((2 m+1)^{2}\right)$ common pairs.

Lemma 3.6. Let $V$ be a set of cardinality $4 m+2$. Constructing two $\left((2 m+1)^{2}\right)$ rounds of $V$ having $\sigma\left((2 m+1)^{2}\right)$ common pairs is equivalent to constructing two blocks (subsets) of $V$, say $A$ and $B$, both of cardinality $2 m+1$, such that

$$
|A \cap B|=m \text { or } m+1 \text {. }
$$

Proof. Clearly, if $\Re_{1}$ and $\Re_{2}$ are $\left((2 m+1)^{2}\right)$-rounds containing $\sigma\left((2 m+1)^{2}\right)$ $=2 \mathrm{~m}^{2}$ common pairs, then by Theorem 2.2 , each block of $\Re_{1}$ intersects each block of $\Re_{2}$ in $m$ or $m+1$ elements. Hence, we can take either block of $\Re_{1}$ to be $A$ and either block of $\Re_{2}$ to be $B$.

Conversely, let $A$ and $B$ be blocks of $V$, each of cardinality $2 m+1$, such that $|A \cap B|=m$ or $m+1$. Let $\bar{A}=V \backslash A$ and $\bar{B}=V \backslash B$. It is an easy matter to check that $\Re_{1}=\{A, \bar{A}\}$ and $\Re_{2}=\{B, \bar{B}\}$ are $\left((2 m+1)^{2}\right)$-rounds such that each block of $\Omega_{1}$ intersects each block of $\Re_{2}$ in $m$ or $m+1$ elements. Hence by Lemma $2.2, \Re_{1}$ and $\Re_{2}$ have $\sigma\left((2 m+1)^{2}\right)$ common pairs.

We are now in a position to prove

THEOREM 3.7. If $p^{\alpha}=4 m+3$, where $p$ is a prime and $\alpha$ is a positive integer, then $R\left((4 m+3)^{2}\right) \geqslant 8 m+7$.

Proof. Bose (1947) has established that the set of quadratic residues, say $Q$ of the Galois field of order $4 m+3, G=G F(4 m+3)$, is a difference set (M. Hall, 
Jr. (1967)) of index $m$, as is the set of quadratic nonresidues, say $N$. That is, the multi-set

$$
(a-b \mid a, b \in Q, a \neq b)
$$

contains each element $g \in G \backslash\{0\}$ precisely $m$ times, as does the multi-set $(a-b \mid a, b \in N, a \neq b)$. Furthermore, $Q^{\prime}=Q \cup\{0\}$ and $N^{\prime}=N \cup\{0\}$ are both difference sets of index $m+1$.

For any $g \in G$ and for any subset $S \subseteq G$, define

$$
S+g=\{s+g \mid s \in S\} \text {. }
$$

Now, for $a \in Q$ and $g \in G \backslash\{0\}, a \in Q \cap(Q+g)$ if and only if there is an element $b \in Q$ such that $a=b+g$ or, equivalently, $a-b=g$. Since $Q$ is a difference set of index $m$, it follows that, for any $g \in G \backslash\{0\},|Q \cap(Q+g)|=m$ and hence, for any $\{g, h\} \subseteq G, g \neq h$,

$$
|(Q+g) \cap(Q+h)|=m \text {. }
$$

Similarly, for any $\{g, h\} \subseteq G, g \neq h,|(N+g) \cap(N+h)|=m, \mid\left(Q^{\prime}+g\right) \cap$ $\left(Q^{\prime}+h\right) \mid=m+1$, and $\left|\left(N^{\prime}+g\right) \cap\left(N^{\prime}+h\right)\right|=m+1$.

We make use of the following notation. For $i \in\{1,2\}$ and for any subset $S \subseteq G$, let $S_{i}=S \times\{i\}$ : furthermore, for any $g \in G$, let $g_{i}=(g, i) \in G \times\{i\}$. For any $g_{i} \in G_{i}$ and any $h \in G$, define

$$
g_{i}+h=(g+h)_{i}
$$

and, for any subset $S \subseteq G_{1} \cup G_{2}$, define $S+h=\{s+h \mid s \in S\}$.

Now, let $E=Q_{1}^{\prime} \cup Q_{2}$ and let $F=N_{1}^{\prime} \cup Q_{2}$. Then, for $\{g, h\} \subset G, g \neq h$,

$$
\begin{aligned}
|(E+g) \cap(E+h)| & =\left|\left(Q_{1}^{\prime}+g\right) \cap\left(Q_{1}^{\prime}+h\right)\right|+\left|\left(Q_{2}+g\right) \cap\left(Q_{2}+h\right)\right| \\
& =(m+1)+m=2 m+1 .
\end{aligned}
$$

Similarly, $|(F+g) \cap(F+h)|=2 m+1$. Also,

$$
\begin{aligned}
|(E+g) \cap(F+h)| & =\left|\left(Q_{1}^{\prime}+g\right) \cap\left(N_{1}^{\prime}+h\right)\right|+\left|\left(Q_{2}+g\right) \cap\left(Q_{2}+h\right)\right| \\
& =\left|\left(Q_{1}^{\prime}+g\right) \backslash\left(Q_{1}+h\right)\right|+m \\
& =\left|\left\{g_{1}\right\} \backslash\left(Q_{1}+h\right)\right|+\left|\left(Q_{1}+g\right) \backslash\left(Q_{1}+h\right)\right|+m \\
& =\left|\left\{g_{1}\right\} \backslash\left(Q_{1}+h\right)\right|+2 m+1 .
\end{aligned}
$$

Now $g_{1} \in Q_{1}+h$ if and only if $g-h \in Q$. Hence,

$$
|(E+g) \cap(F+h)|= \begin{cases}2 m+1 & \text { if } g-h \in Q \\ 2 m+2 & \text { if } g-h \in N\end{cases}
$$


For any $g \in G$,

$$
\begin{aligned}
|(E+g) \cap(F+g)| & =\left|\left(Q_{1}^{\prime}+g\right) \cap\left(N_{1}^{\prime}+g\right)\right|+\left|\left(Q_{2}+g\right) \cap\left(Q_{2}+g\right)\right| \\
& =(1)+(2 m+1)=2 m+2 .
\end{aligned}
$$

Finally,

$$
\left|G_{1} \cap(E+g)\right|=\left|G_{1} \cap(F+g)\right|=2 m+2 .
$$

Thus, we have shown that

$$
\mathscr{B}=\bigcup_{g \in G}\{E+g, F+g\} \cup\left\{G_{1}\right\}
$$

is a set of $8 m+7$ blocks of $G_{1} \cup G_{2}$ such that any two of these blocks intersect in $2 m+1$ or $2 m+2$ elements. By Lemma 3.6, there exist $8 m+7\left((4 m+3)^{2}\right)$ rounds of $G_{1} \cup G_{2}$ having $\sigma\left((4 m+3)^{2}\right)$ common pairs and hence $\Re\left((4 m+3)^{2}\right)$ $\geqslant 8 m+7$, as required.

We conclude this section by observing that Lemma 3.3 and Theorem 3.7 imply

$$
R\left((4 m+3)^{2}\right) \geqslant 8 m+7
$$

for some integers $4 m+3$ which are not primes nor powers of primes, for example, when $4 m+3 \in\{15,39,55,63\}$.

\section{4. $\sigma(m n)$ and $R(m n)$}

In this section, we continue our investigation of $\sigma(G, H)$ and $R(G, H)$ using the language of combinatorial designs.

Let $k_{1}, k_{2}, \ldots, k_{s}$ be distinct positive integers and let $i_{1}, i_{2}, \ldots, i_{s}$ be positive integers. Let

$$
\sum_{j=1}^{s} i_{j}=t \text { and } \sum_{j=1}^{s} i_{j} k_{j}=v
$$

For

$$
r=k_{1}^{i_{1}} k_{2}^{i_{2}} \cdots k_{s}^{i_{s}},
$$

a $k_{1}^{i_{1}} k_{2}^{i_{2}} \cdots k_{s}^{i_{s}}$-round, or more briefly, an $r$-round, say $\Re=\left\{B_{1}, B_{2}, \ldots, B_{t}\right\}$, is a partition of some $v$-set, say $V$, into $t$ blocks such that $i_{1}$ of the blocks have cardinality $k_{1}, i_{2}$ have cardinality $k_{2}$, and so on until $i_{s}$ have cardinality $k_{s}$. As in Section 3, the pairs of $\Re$ are all the pairs $\{a, b\} \subseteq V, a \neq b$, such that, for some block $B \in \Re,\{a, b\} \subseteq B$. We define $\sigma\left(k_{1}^{i_{1}} k_{2}^{i_{2}} \cdots k_{s}^{i_{s}}\right)=\sigma(r)$ to be the largest integer such that any two $r$-rounds of $V$ contain at least $\sigma(r)$ common pairs and 
define $R(r)$ to be the largest integer $l$ such that there are $l r$-rounds, say $\mathscr{R}_{1}, \Re_{2}, \ldots, \Re_{1}$, any two of which have $\sigma(r)$ common pairs. If no such largest integer $l$ exists, then we say $R(r)=\infty$.

In this section we study $R(r)$ in the special case where $r=m^{1} n^{1}$ or more simply, $r=m n$. Since we have considered $r=m^{2}$ in Section 3, we further assume that $m>n$.

LEMMA 4.1. (i) For $m \geqslant 3 n-1$, two mn-rounds have $\sigma(m n)$ common pairs if and only if the two blocks of size $m$ intersect in $p=m-n$ elements.

For $n<m<3 n-1$, let $p=[(3 m-n+1) / 4]$, the integer part of $(3 m-n+$ 1)/4. Two mn-rounds have $\sigma(\mathrm{mn})$ common pairs if and only if the two blocks of size $m$

(ii) intersect in $p$ elements when $m+n \neq 2(\bmod 4)$ and

(iii) intersect in $p$ or $p+1$ elements when $m+n \equiv 2(\bmod 4)$.

Proof. Let one round consist of blocks $A$ and $\bar{A}$ and another of $B$ and $\bar{B}$, where $|A|=|B|=m$ and $|\bar{A}|=|\bar{B}|=n$; furthermore, let $|A \cap B|=x$. Then $|A \cap \bar{B}|=m-x,|B \cap \bar{A}|=m-x$ and $|\bar{A} \cap \bar{B}|=n-(m-x)=n-m+x$. Clearly, $n-m+x \geqslant 0$ or $x \geqslant m-n$ : hence, $m-n \leqslant x \leqslant m$.

The number of pairs common between these two rounds is given by

$$
\begin{aligned}
N(x) & =\left(\begin{array}{l}
x \\
2
\end{array}\right)+\left(\begin{array}{c}
m-x \\
2
\end{array}\right)+\left(\begin{array}{c}
m-x \\
2
\end{array}\right)+\left(\begin{array}{c}
n-m+x \\
2
\end{array}\right) \\
& =2 x^{2}+(n-3 m) x+\frac{3 m^{2}-2 m n+n^{2}-m-n}{2} .
\end{aligned}
$$

It can readily be shown that $N(x)$ has a minimum at $x=(3 m-n) / 4$ and that it is symmetric about the line $x=(3 m-n) / 4$.

If $m-n \geqslant(3 m-n-1) / 4$, or equivalently, if $m \geqslant 3 n-1$, then $N(x)$ is strictly increasing for integers $x$ in the admissible interval $m-n \leqslant x \leqslant m$; hence, $\sigma(m n)=N(m-n)$. This establishes (i).

Otherwise, $n<m<3 n-1$. Since $x=|A \cap B|$ is an integer, it follows that $\sigma(m n)=N(p)$ where $p=[(3 m-n+1) / 4]$, the integer part of $(3 m-n+1) / 4$. For $m+n \neq 2(\bmod 4), N(p)<N(q)$ for any integer $q \neq p$, and, for $m+n \equiv$ $2(\bmod 4), N(p)=N(p+1)$ and, for any integer $q \notin\{p, p+1\}, N(p)<N(q)$. Hence, we have parts (ii) and (iii).

Before stating a useful corollary of this lemma we require a definition. An $(r, \lambda)$-design is a pair $(X, \mathcal{Q})$ where $X$ is a set and $\mathcal{Q}$ is a collection of (not necessarily distinct) subsets of $X$, called blocks, with the following two properties: (i) any element of $X$ is contained in precisely $r$ blocks of $Q$ and (ii) any pair of 
distinct elements of $X$ is contained in precisely $\lambda$ blocks of $\mathcal{Q}$. Note that blocks of cardinality one are permitted.

COROLlaRY 4.2. If $m+n \neq 2(\bmod 4)$, then the existence of $t$ mn-rounds, each pair of them having $\sigma(\mathrm{mn})$ common pairs, is equivalent to the existence of an $(r, \lambda)$-design, $(X, \mathbb{Q})$, with $(r, \lambda)=(m, p),|X|=t$ and $|\mathbb{Q}| \leqslant m+n$.

Proof. Let there be $t m n$-rounds of the $(m+n)$-set such that any two of these $m n$-rounds have $\sigma(m n)$ common pairs. Let $B_{1}, B_{2}, \ldots, B_{t}$ be the blocks of cardinality $m$ from these $t m n$-rounds and let $V=B_{1} \cup B_{2} \cup \cdots \cup B_{t}$. The dual (see, for example, M. Hall, Jr. (1967)) of the design $\left(V,\left\{B_{1}, B_{2}, \ldots, B_{t}\right\}\right)$ is an $(r, \lambda)$ design, say $(X, \mathscr{Q})$, having $(r, \lambda)=(m, p),|X|=t$ and $|\mathcal{Q}| \leqslant m+n$.

It follows readily that the converse also holds.

Since Fisher's inequality (M. Hall, Jr. (1967)) holds for $(r, \lambda)$-designs, we immediately have

LEMMA 4.3. $R(m n) \leqslant m+n$, provided $m+n \neq 2(\bmod 4)$.

For certain values of the parameters $m$ and $n$, we can obtain a sharper upper bound.

$$
\begin{aligned}
& \text { LEMMA 4.4. If } p(m+n-1) \neq m(m-1) \text {, and } m+\neq 2(\bmod 4) \text { then } \\
& \qquad R(m n)<m+n .
\end{aligned}
$$

Proof. By hypothesis, $p(m+n-1) \neq m(m-1)$. To obtain a contradiction, assume $R(m n)=m+n$. By Lemma 4.2, there exists an $(r, \lambda)$-design, with $(r, \lambda)=(m, p)$, say $(X, \mathbb{Q})$, having $|X|=m+n=|\mathbb{Q}|$. Ryser (1950) has shown that such an $(r, \lambda)$-design is, in fact, a symmetric balanced incomplete block design with parameters $(v, b, r, k, \lambda)=(m+n, m+n, m, m, p)$ and hence $\lambda(v-1)=r(k-1)$ or $p(m+n-1)=m(m-1)$. From this contradiction, we conclude that $R(m n) \neq m+n$, which, by Lemma 4.3, implies $R(m n)<m+n$.

Obviously, if $A$ and $B$ are two $m$-subsets of an $(m+n)$-set which intersect in $p$ elements, then their complements, $\bar{A}$ and $\bar{B}$, are two $n$-subsets which intersect in $n-m+p$ elements. Hence, it is easy to determine $R(m n)$ when the $m$-subsets intersect in $m-n$ elements: in this case, $R(m n)$ is the maximum number of disjoint $n$-subsets that can be selected from an $(m+n)$-set. Now by Lemma 4.1(i), the $m$-subsets of two $m n$-rounds having $\sigma(m n)$ common pairs intersect in $m-n$ elements whenever $m \geqslant 3 n-1$. Thus we have 
LEMMA 4.5. If $m \geqslant 3 n-1$, then

$$
R(m n)=[(m+n) / n],
$$

the integer part of $(m+n) / n$.

It now follows from the above lemma that $R(m 1)=m+1$.

We now consider the case where $n=m-1$. For results on Hadamard matrices, the reader is referred to Wallis, Street, Wallis (1972). Note that in this case $m+n \geq 2(\bmod 4)$.

THEOREM 4.6. If there is a Hadamard matrix of order $4 k$, then

$$
R((2 k)(2 k-1))=4 k-1 \text {. }
$$

Proof. The existence of a Hadamard matrix of order $4 n$ implies the existence of a symmetric balanced incomplete block design with parameters $(v, b, r, k, \lambda)$ $=(4 k-1,4 k-1,2 k, 2 k, k)$ (M. Hall, Jr. (1967)). Hence, by Corollary 4.2, $R(r) \geqslant 4 k-1$ and, consequently, $R=4 k-1$, as required.

We next establish a lower bound for $R((2 k+1)(2 k))$.

LEMMA 4.7. If there is an Hadamard matrix of order $4 k$, then

$$
R((2 k+1)(2 k)) \geqslant 4 k-1 \text {. }
$$

Proof. The existence of a Hadamard matrix of order $4 k$ is equivalent to the existence of a symmetric balanced, incomplete block design with parameters $(v, b, r, k, \lambda)=(4 k-1,4 k-1,2 k-1,2 k-1, k-1)$ (M. Hall, Jr. (1967)). Adjoin two addditional blocks, both containing all $4 k-1$ elements. We obtain an $(r, \lambda)$-design $(X, \mathbb{Q})$ having $(r, \lambda)=(2 k+1, k+1),|X|=4 k-1$ and $|\mathbb{Q}|$ $=4 k+1$. Hence, by Corollary $4.2, R \geqslant 4 k-1$.

For $p=p((2 k+1)(2 k)), \quad p(m+n-1)=(k+1) 4 k \neq(2 k+1) 2 k$ $=m(m-1)$ and, consequently, by Lemma $4.4, R((2 k+1)(2 k))<4 k+1$. Thus we have

THEOREM 4.8. If there is a Hadamard matrix of order $4 k$ then,

$$
4 k-1 \leqslant R((2 k+1)(2 k)) \leqslant 4 k .
$$

The following four $3^{1} 2^{1}$-rounds demonstrate that $R\left(3^{1} 2^{1}\right)=4$ :

$\begin{array}{ll}123 & 45 \\ 124 & 35 \\ 234 & 15 \\ 134 & 25\end{array}$


Also, $R\left(4^{1} 2^{1}\right)=15$ by Lemma 4.13 below. These results, together with Lemma 4.5, immediately determine $R\left(m^{1} 2^{1}\right) . R\left(3^{1} 2^{1}\right)=4$ and $R\left(4^{1} 2^{1}\right)=15$ : for $m>4$, $m \geqslant 3 n-1=4$ which implies $R=[(m+2) / 2]$, the integer part of $(m+2) / 2$.

We conclude this section by considering $R(m(m-2))$. We investigate the cases $R((2 k+1)(2 k-1))$ and $R((2 k+2)(2 k))$ separately.

\section{LEMMA 4.9. $R((2 k+1)(2 k-1)) \geqslant R((2 k)(2 k-1))$.}

Proof. Let $R((2 k)(2 k-1))=t$. By Lemma 4.1, $p=k$, and by Corollary 4.2, there is a $(2 k, k)$-design $(X, \mathbb{Q})$, having $|X|=t$ and $|\mathscr{Q}| \leqslant 4 k-1$. Let $\mathbb{Q}^{\prime}=\mathbb{Q} \cup$ $\{X\}$. Then $\left(X, \mathbb{Q}^{\prime}\right)$ is a $(2 k+1, k+1)$-design having $|X|=t$ and $\left|Q^{\prime}\right|=4 k$ which, by Corollary 4.2 , implies that

$$
R((2 k+1)(2 k-1)) \geqslant t=R((2 k)(2 k-1)) .
$$

THEOREM 4.10. If there is a Hadamard matrix of order $4 k$, then

$$
R((2 k+1)(2 k-1))=4 k-1 .
$$

Proof. By Lemma 4.4, $R((2 k+1)(2 k-1))<4 k$, and by Lemma 4.6 and 4.9 , $R \geqslant 4 k-1$.

We now consider $R((2 k+2)(2 k))$. In this case, $m+n=4 k+2 \equiv 2(\bmod 4)$ and Lemma 4.1(iii) implies that two $(2 k+2)(2 k)$-rounds have $\sigma$ common pairs if and only if blocks of size $2 k+2$ intersect in $k+1$ or $k+2$ elements, or equivalently, if and only if the blocks of size $2 k$ intersect in $k$ or $k-1$ elements. Thus we have

LEMMA 4.11. $R((2 k+2)(2 k))$ is equal to the number of $2 k$-subsets which may be selected from a $4 k+2$-set such that any two of the $2 k$-subsets intersect in $k$ or $k-1$ elements.

LEMMA 4.12. $R((2 k+2)(2 k)) \leqslant 4 k+5$ provided $k \geqslant 4$ and

$$
R\left(4^{1} 2^{1}\right) \leqslant 15, \quad R\left(6^{1} 4^{1}\right) \leqslant 15, \quad R\left(8^{1} 6^{1}\right) \leqslant 18 .
$$

PROOF. This follows directly from Theorem 2.3 when we assume

$$
\begin{aligned}
& E=\left(\begin{array}{c}
4 k+2 \\
2
\end{array}\right), \quad e=\left(\begin{array}{c}
2 k+2 \\
2
\end{array}\right)+\left(\begin{array}{c}
2 k \\
2
\end{array}\right), \\
& \theta=\left(\begin{array}{c}
k+2 \\
2
\end{array}\right)+3\left(\begin{array}{l}
k \\
2
\end{array}\right)=3\left(\begin{array}{c}
k+1 \\
2
\end{array}\right)+\left(\begin{array}{c}
k-1 \\
2
\end{array}\right) .
\end{aligned}
$$


THEOREM 4.13. $R\left(4^{1} 2^{1}\right)=15$.

Proof. By virtue of Lemma 4.12, it is enough to construct 15 blocks of size 2 such that any two of them intersect in 0 or 1 element. This is easily done by taking all $\left(\begin{array}{l}6 \\ 2\end{array}\right)=15$ pairs from the 6 elements.

Now we look to get some lower bounds for the $R((2 k+2)(2 k))$ value. In this context we use the following result of Bose.

Lemma 4.14 (1947). Let $p^{\alpha}=4 k+1$ where $p$ is a prime. Then among the totality of differences of the quadratic residues of $G F\left(p^{\alpha}\right)$, every quadratic residue occurs $(k-1)$ times and every quadratic nonresidue occurs $k$ times.

LEMMA 4.15. If $4 k+1$ is a prime power, then $R((2 k+2)(2 k)) \geqslant 4 k+1$.

Proof. Let the element-set be $G F(4 k+1) \cup\{\infty\}$ and let $x$ be a primitive element of $G F(4 k+1)$. The set of quadratic residues is

$$
B=\left\{x^{0}, x^{2}, \ldots, x^{4 k-2}\right\} .
$$

Consider the set of blocks $\{B+y: y \in G F(4 k+1)\}$. It follows from Lemma 4.14 that

$$
|(B+y) \cap(B+w)|= \begin{cases}k-1 & \text { if } y-w \text { is a quadratic residue } \\ k & \text { if } y-w \text { is a non-residue. }\end{cases}
$$

Hence, Lemma 4.11 implies $R((2 k+2)(2 k)) \geqslant 4 k+1$.

Lemma 4.16. $R[(4 k+4)(4 k+2)] \geqslant 8 k+7$ provided $4 k+3$ is a prime power.

Proof. We apply the same technique that we use in proving Theorem 3.7. Let $G$ be the Galois field with $4 k+3$ elements and let

$$
G_{i}=G \times\{i\}, \quad i=1,2 .
$$

Our element set is $G_{1} \cup G_{2}$ so that $\left|G_{1} \cup G_{2}\right|=8 k+6$.

$Q$ is the set of quadratic residues in $G$ and $N$ is the set of quadratic non-residues in $G$. Let $E=Q_{1} \cup Q_{2}$ and $F=N_{1} \cup Q_{2}$. Then, as in Theorem 3.7, one can show that

$$
B=\bigcup_{g \in G}\{E+g, F+g\} \cup\left(G_{1} \backslash\left\{0_{1}\right\}\right)
$$


is a set of $8 k+7$ blocks of $G_{1} \cup G_{2}$ such that any two of these blocks intersect in $2 k$ or $2 k+1$ elements. Hence Lemma 4.11 implies

$$
R[(4 k+4)(4 k+2)] \geqslant 8 k+7 .
$$

LEMMA 4.17. $R((4 k+2)(4 k)) \geqslant 2 \min \left\{R((2 k+2)(2 k)), R\left((2 k)^{2}\right)\right\}+1$.

Proof. Let $\min \left\{R((2 k+2)(2 k)), R\left((2 k)^{2}\right)\right\}=t$. Let $X$ be a set with $4 k+2$ elements. Then there are $t$ subsets of $X$ say, $B_{1}, B_{2}, \ldots, B_{t}$, such that $\left|B_{i}\right|=2 k$ and $\left|B_{i} \cap B_{j}\right|=k$ or $k-1$ for all $i, j=1, \ldots, t$. Also let $Y$ be a set with $4 k$ elements such that $Y \cap X=\varnothing$. Then there are $t$ subsets of $Y$, say, $C_{1}, C_{2}, \ldots, C_{t}$, such that $\left|C_{i}\right|=2 k$ and $\left|C_{i} \cap C_{j}\right|=k$ for all $i, j=1,2, \ldots, t$. Now let

$$
\begin{aligned}
D_{i} & =B_{i} \cup C_{i}, & i & =1, \ldots, t, \\
E_{i} & =B_{i} \cup \bar{C}_{i}, & i & =1, \ldots, t \text { where } \bar{C}_{i}=Y \backslash C_{i} .
\end{aligned}
$$

Then

$$
\bigcup_{i=1}^{t}\left\{D_{i}, E_{i}\right\} \cup Y
$$

is a set of $2 t+1$ blocks of $X \cup Y$ such that each has cardinality $4 k$ and any two of these blocks intersect in $2 k$ or $2 k-1$ elements. 1.

LEMMA 4.18. $R[(4 k+4)(4 k+2)] \geqslant 2 \min \left\{R((2 k+2)(2 k)), R\left((2 k+2)^{2}\right)\right\}+$

Proof. Let $\min \left\{R((2 k+2)(2 k)), R\left((2 k+2)^{2}\right)\right\}=t$. Let $X$ be a set having $4 k+2$ elements. Then, there are $t$ subsets of $X$, say, $B_{1}, B_{2}, \ldots, B_{t}$, such that $\left|B_{i}\right|=2 k$ and $\left|B_{i} \cap B_{j}\right|=k$ or $k-1$ for all $i, j=1,2, \ldots, t$. Also, let $Y$ be a set having $4 k+4$ elements, $Y \cap X=\varnothing$. Then, there are $t$ subsets of $Y$, say, $C_{1}, C_{2}, \ldots, C_{t}$ such that $\left|C_{i}\right|=2 k+2$ and $\left|C_{i} \cap C_{j}\right|=k+1$ for all $i, j=$ $1,2, \ldots, t$. Now let

$$
\begin{aligned}
& D_{i}=B_{i} \cup C_{i}, \quad i=1,2, \ldots, t, \\
& E_{i}=B_{i} \cup \bar{C}_{i}, \quad i=1,2, \ldots, t \quad \text { where } \bar{C}_{i}=Y \backslash C_{i} .
\end{aligned}
$$

Then

$$
\bigcup_{i=1}^{t}\left\{D_{i}, E_{i}\right\} \cup Y
$$

is a set of $2 t+1$ blocks of $X \cup Y$ such that each has cardinality $4 k+2$ and any two of them intersect in $2 k$ or $2 k+1$ elements.

Hence, the result follows by Lemma 4.11 . 


\section{Double-cones}

In this section we consider $\sigma(G, H)$ and $R(G, H)$ where $H$ is a complete graph and $G$ is a graph having $\nu(G)=\nu(H)$. In the preceding sections, we have considered the case where $G$ is the union of disjoint complete subgraphs of $\mathrm{H}$. Nemeth (1976) has considered the cases where $G$ is a star, a cycle, a wheel, a rim-deleted wheel, and a spoke-deleted wheel. We now consider the case where $G$ is a double-cone.

A double-cone on $n+2$ vertices consists of a circuit of length $n$, together with two independent vertices, say $s$ and $t$, which are adjacent to each vertex of the circuit. Observe that $s$ and $t$ each have valence $n$ and all the other vertices have valence 4 . We call the circuit of length $n$ the rim of the double-cone and, for $n>4$, the rim is uniquely determined by the vertices of valence 4 . We will represent a double-cone as shown below.

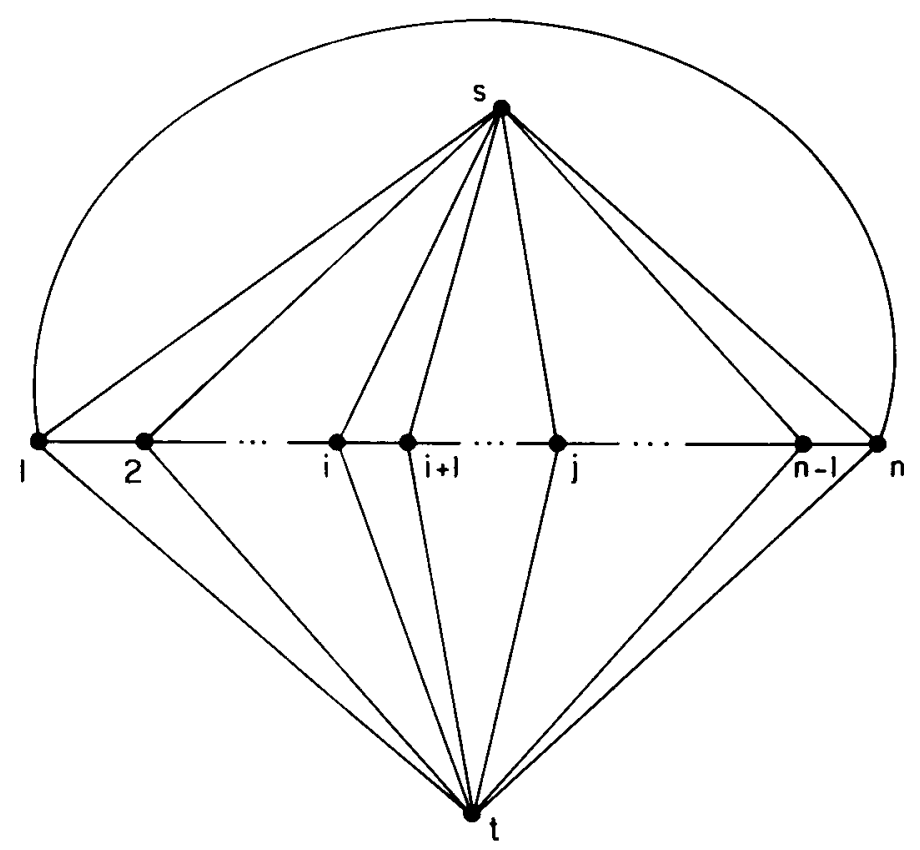

THEOREM 5.1. Let $H$ be a complete graph and let $G$ be a double-cone (having $\nu(G)=\nu(H))$. For $\nu(H) \geqslant 8, \sigma=\sigma(G, H)=8$ and $R=R(G, H)=2$.

Proof. We begin by showing $\sigma=8$. 
Let $G$ be a subgraph of $H$ which is a double-cone and let $G^{\prime}$ be a subgraph of $H$ isomorphic to $G$. Label the vertices of $H$ with the symbols of $\{1,2, \ldots, n\} \cup\{s, t\}$ so that the rim of $G$ has $1,2, \ldots, n$ as its vertices.

If the isomorphism $\theta: G \rightarrow G^{\prime}$ maps either of $s$ or $t$ into $\{s, t\}$, then it can be shown that $\varepsilon\left(G \cap G^{\prime}\right) \geqslant n+3 \geqslant 9$. Hence, we consider an isomorphism $\theta: G \rightarrow G^{\prime}$ such that

$$
\theta(s)=k, \quad \theta(t)=l, \quad \theta^{-1}(s)=i, \quad \theta^{-1}(t)=j
$$

where $\{i, j, k, l\} \subseteq\{1,2, \ldots, n\}$.

Case 1. Vertices $i$ and $j$ are not adjacent in $G$.

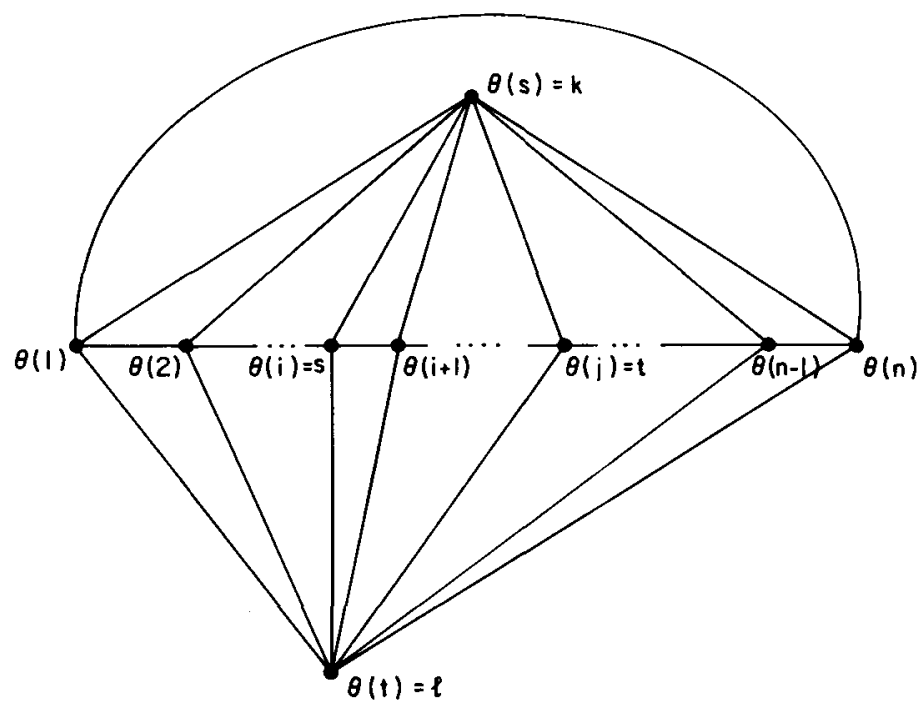

Clearly the 4 edges on $s$ and the 4 edges on $t$ in $G^{\prime}$ are also in $E(G)$; furthermore, at least one additional edge on $k$ in $G^{\prime}$ and at least one additional edge on $l$ in $G^{\prime}$ are in $E(G)$. Therefore $\varepsilon\left(G \cap G^{\prime}\right) \geqslant 10$.

Case 2. Vertices $k$ and $l$ are not adjacent in $G$. As in Case 1 , it can be shown that $\varepsilon\left(G \cap G^{\prime}\right) \geqslant 10$.

Case 3. Vertices $i$ and $j$ are adjacent in $G$ and vertices $k$ and $l$ are adjacent in $G$. 


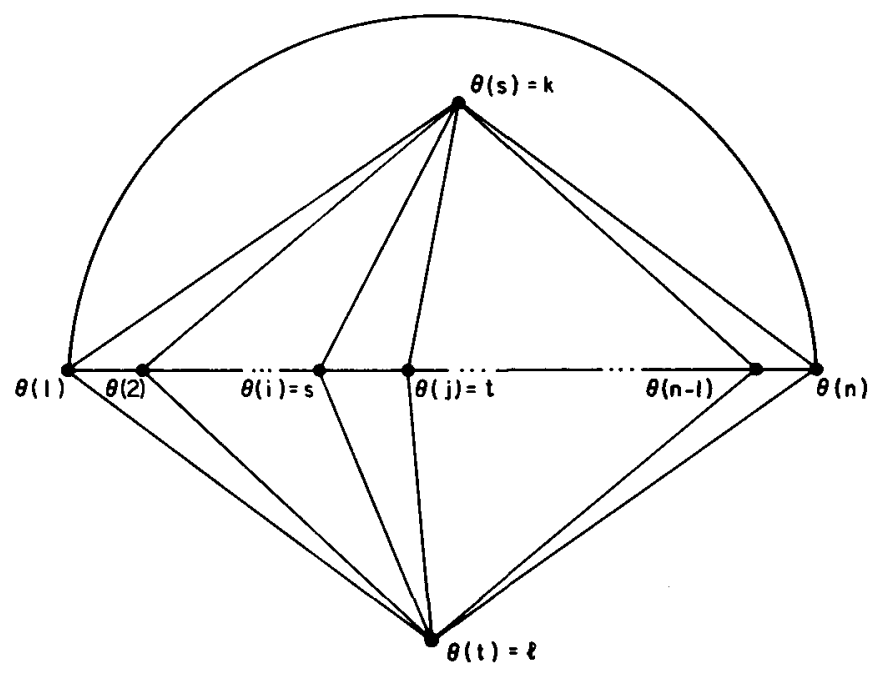

Then three of the edges incident with $s$ in $G^{\prime}$ are in $E(G)$, as are three of the edges incident with $t$. Precisely one additional edge incident with $k$ in $G^{\prime}$ is in $E(G)$, as is one additional edge on $l$ in $G$. Hence, $\varepsilon\left(G \cap G^{\prime}\right) \geqslant 8$. In addition, if the rims of $G$ and $G^{\prime}$ are edge disjoint, then it follows that $\varepsilon\left(G \cap G^{\prime}\right)=8$. If $\nu(H) \geqslant 8$, then it is possible to obtain $G$ and $G^{\prime}$ such that their rims are edge-disjoint. This can be shown by Dirac's Theorem on Hamiltonian circuits (Bondy \& Murty (1976)). Thus $\sigma=8$.

To obtain a contradiction, suppose $G^{\prime \prime}$ is a third subgraph of $H$ isomorphic to $G$ such that any pair of $G, G^{\prime}, G^{\prime \prime}$ have precisely 8 edges in common. By the above argument, $s$ and $t$ must be adjacent on the rims of $G^{\prime}$ and $G^{\prime \prime}$. Since the rims of $G^{\prime}$ and $G^{\prime \prime}$ are not edge-disjoint, $\varepsilon\left(G^{\prime} \cap G^{\prime \prime}\right)>8$, a contradiction. Hence, $R=2$.

LEMMA 5.2. If $D_{n}$ represents a double-cone on $n$ vertices, then

$$
\begin{array}{lll}
\sigma\left(D_{5}, K_{5}\right)=8 & \text { and } & R\left(D_{5}, K_{5}\right)=10, \\
\sigma\left(D_{6}, K_{6}\right)=9 & \text { and } & R\left(D_{6}, K_{6}\right)=5, \\
\sigma\left(D_{7}, K_{7}\right)=9 & \text { and } & R\left(D_{7}, K_{7}\right)=3 .
\end{array}
$$

\section{References}

[1] J. A. Bondy and U. S. R. Murty (1976), Graph theory with applications (Macmillan Press, Hong Kong).

[2] R. C. Bose (1947), 'On resolvable series of balanced incomplete block designs', Sankhya 8. $251-257$. 
[3] C. M. Cordes (1978), 'A new type of combinatorial design', J. Combinatorial Theory, Ser. A. 24, 251-257.

[4] A. Hartman, R. C. Mullin and D. R. Stinson (1980), Exact covering configurations and Steiner systems, Research Report CORR 80-40 (Dept. of Combinatorics and Optimization, University of Waterloo, Waterloo, Ontario, Canada).

[5] M. Hall, Jr. (1967), Combinatorial theory (Blaisdell Publishing Co., Wallham, Mass).

[6] S. M. Johnson (1962), 'A new upper bound for error-correcting codes', IRE Trans. Informat. Theory 8, 203-207.

[7] D. McCarthy and G. H. J. van Rees (1977), 'Some results on a combinatorial problem of Cordes', J. Austral. Math. Soc. Ser. A. 23, 439-452.

[8] R. C. Mullin and R. G. Stanton (1978), 'A characterization of pseudo-affine designs and their relation to a problem of Cordes', Ann. Discrete Math. 2. 231-238.

[9] E. Nemeth (1976), 'A note on a minimax problem in scheduling', Proceedings 6 th Manitoba Conference on Numerical Math., pp. 343-349 (Winnipeg, Manitoba).

[10] H. J. Ryser (1950), 'A note on a combinatorial problem', Proc. Amer. Math. Soc. 1, 422-424.

[11] W. D. Wallis, A. P. Street and J. S. Wallis (1972), Combinatorics: Room squares, sum-free sets, Hadamard matrices, Lecture Notes in Mathematics, no. 292 (Springer-Verlag, Berlin).

\section{University of Waterloo}

Waterloo, Ontario

Canada 